\title{
Supplementary information
}

\section{Observation of localized vibrational modes of graphene nanodomes by inelastic atom scattering}

D. Maccariello, ${ }^{1,2}$ A. Al Taleb, ${ }^{2}$ F. Calleja, ${ }^{1}$ A. L. Vázquez de Parga,,${ }^{1,2,3,4}$ P. Perna, ${ }^{1}$ J. Camarero, ${ }^{1,2,3,4}$ E. Gnecco, ${ }^{1}$ D. Farías, ${ }^{2,3,4}$ and R. Miranda ${ }^{1,2,3,4}$

${ }^{1}$ Instituto Madrileño de Estudios Avanzados en Nanociencia (IMDEA-Nanociencia), 28049 Madrid, Spain

${ }^{2}$ Departamento de Física de la Materia Condensada, Universidad Autónoma de Madrid, 28049 Madrid, Spain ${ }^{3}$ Instituto de Ciencia de Materiales "Nicolás Cabrera", Universidad Autónoma de Madrid, 28049 Madrid, Spain ${ }^{4}$ Condensed Matter Physics Center (IFIMAC), Universidad Autónoma de Madrid, 28049 Madrid, Spain (Dated: October 1, 2015) 


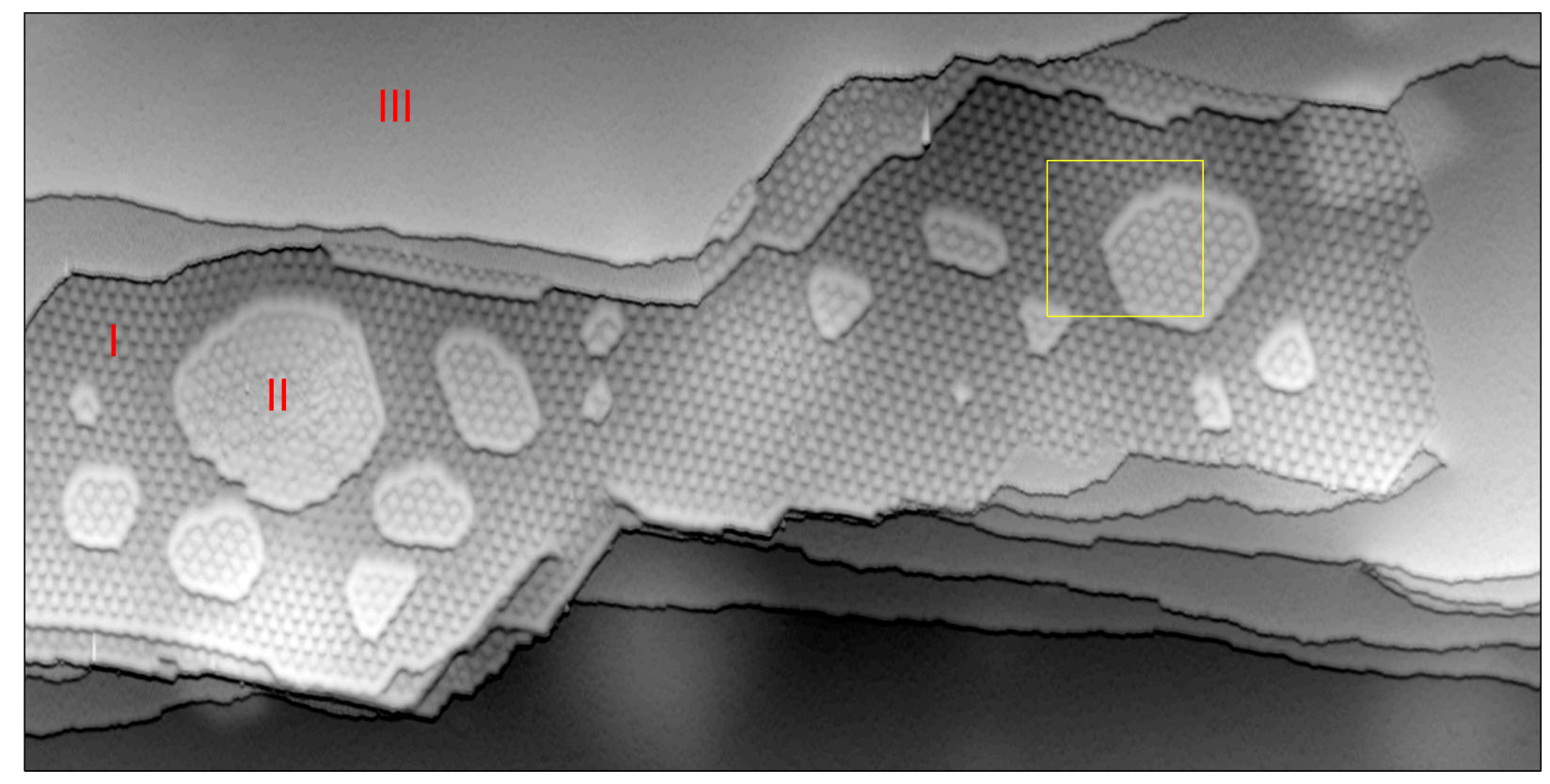

FIG. 1: Early stages of $\mathrm{Cu}$ intercalation. $200 \times 100 \mathrm{~nm}^{2}$ recorded at $80 \mathrm{~K}$. Three areas can be distinguished: I, undisturbed $\mathrm{Gr} / \mathrm{Ru}(0001)$; II, Cu-intercalated $\mathrm{Gr} / \mathrm{Ru}(0001)$; III, pseudomorphic $\mathrm{Cu} / \mathrm{Ru}(0001)$ not covered by graphene. The yellow frame indicates the region where the image of Fig. 1c of main text was acquired. 

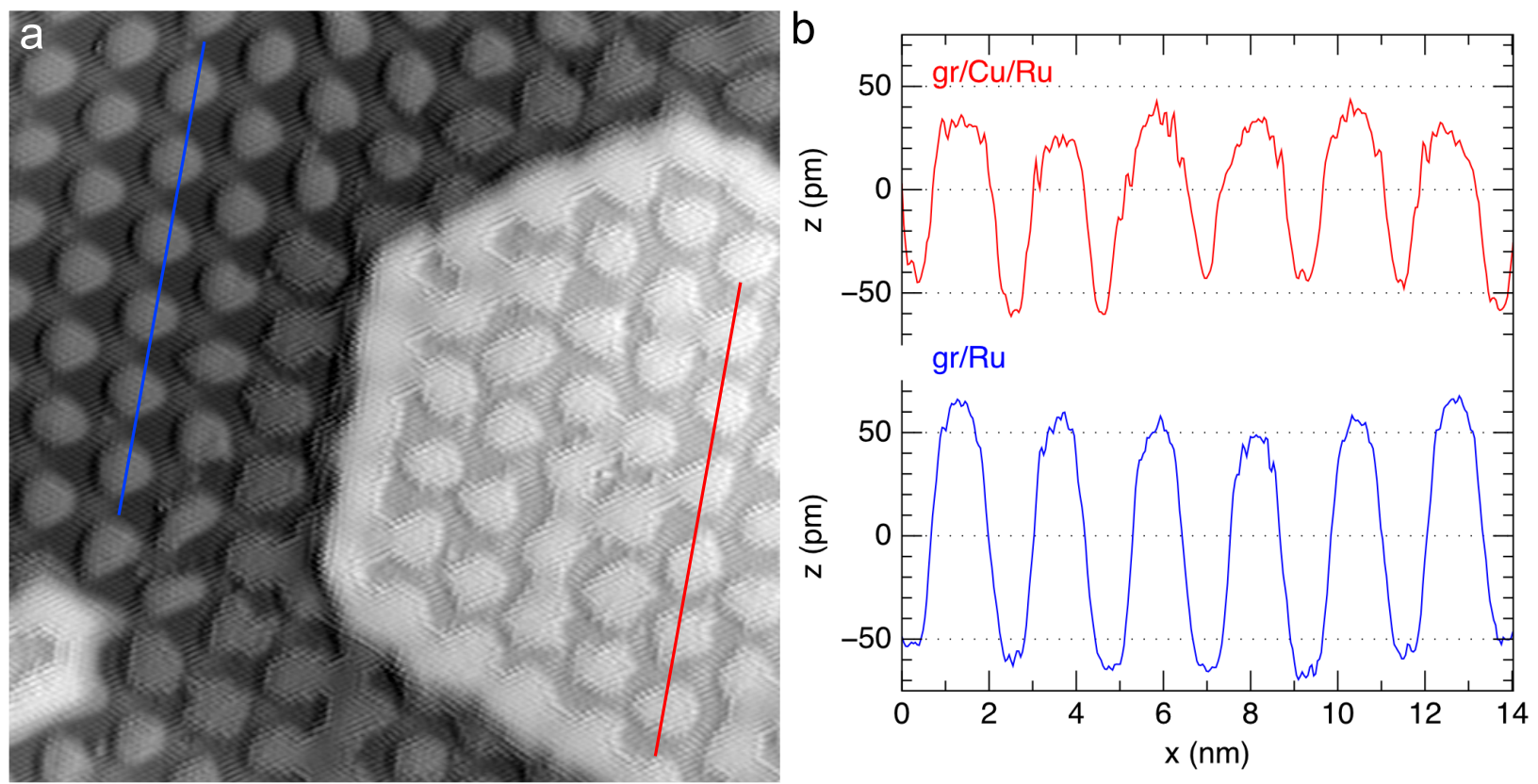

FIG. 2: a) $30 \times 30 \mathrm{~nm}^{2}$ atomic resolution STM image, scanned at $-30 \mathrm{mV}$ and $1 \mathrm{nA}$. b) Topographic profiles along several nanodomes of the $\mathrm{Gr} / \mathrm{Ru}$ (blue) and $\mathrm{Gr} / \mathrm{Cu} / \mathrm{Ru}$ (red) areas, corresponding to the lines shown in the image of panel (a). Average corrugations are (116 \pm 7$) \mathrm{pm}$ for $\mathrm{Gr} / \mathrm{Ru}$ and $(70 \pm 7) \mathrm{pm}$ for $\mathrm{Gr} / \mathrm{Cu} / \mathrm{Ru}$. 


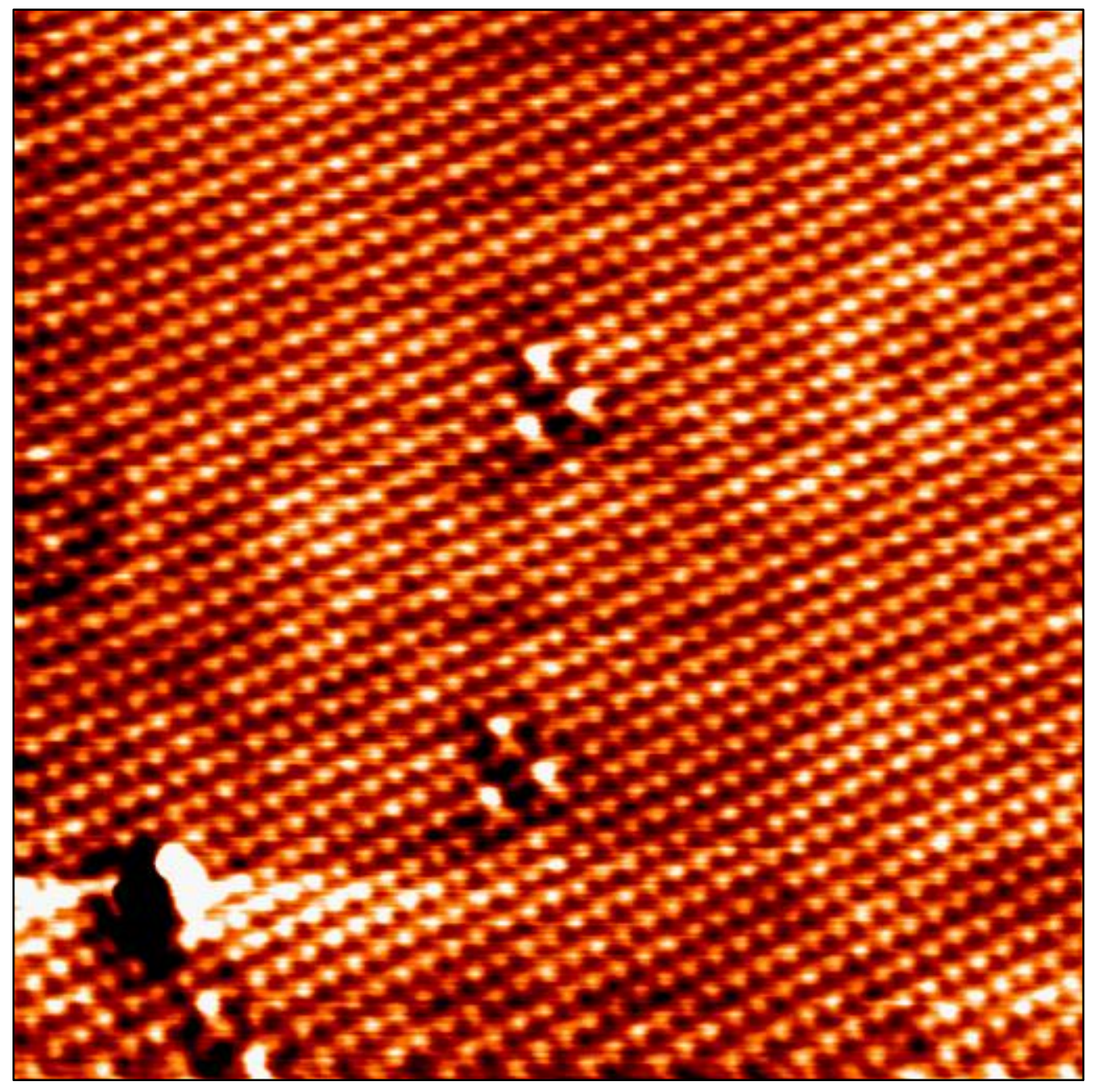

FIG. 3: $10 \times 10 \mathrm{~nm}^{2}$ atomic resolution STM image acquired on $\mathrm{Cu} / \mathrm{Ru}(0001)$. Copper atoms are resolved together with two triangular defects associated to oxygen. The lack of moiré pattern and the size indicate a pseudomorphic ordering with respect to the $\mathrm{Ru}(0001)$ surface. 

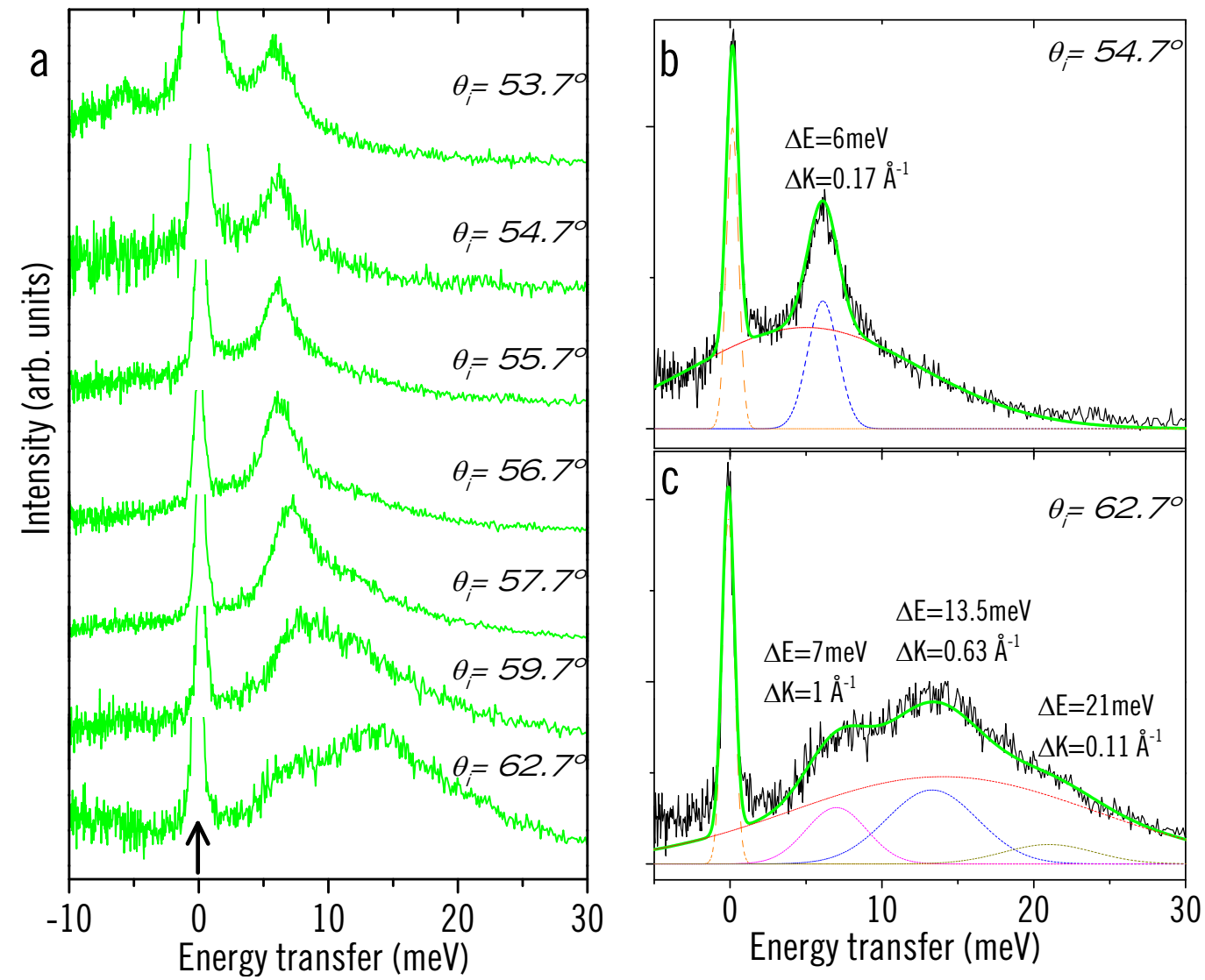

FIG. 4: a) Inelastic HAS spectra measured along $\bar{\Gamma} \overline{\mathrm{M}}$ for the intercalated $\mathrm{Gr} / \mathrm{Cu}(0.4 \mathrm{ML}) / \mathrm{Ru}$ sample at $\mathrm{E}_{\mathrm{i}}=21 \mathrm{meV}$ and $\mathrm{T}_{\mathrm{S}}=300 \mathrm{~K}$ for different incident angles. The arrow indicates the zero of energy scale. b-c) Examples of fits used to identify the number, momentum transfer and energy of the inelastic losses. This sample has regions of intercalated and non-intercalated graphene, and the spectra show a superposition of both contributions. 